Claremont Colleges

Scholarship@ Claremont

All HMC Faculty Publications and Research

HMC Faculty Scholarship

$1-1-1955$

\title{
An Isomorphism Theorem for Real-Closed Fields
}

P. Erdös

L. Gillman

Melvin Henriksen

Harvey Mudd College

\section{Recommended Citation}

Erdös, P.; Gillman, L.; Henriksen, M. An Isomorphism Theorem For Real-Closed Fields. Ann. of Math. (2) 61, (1955). 542-554.

This Article is brought to you for free and open access by the HMC Faculty Scholarship at Scholarship @ Claremont. It has been accepted for inclusion in All HMC Faculty Publications and Research by an authorized administrator of Scholarship @ Claremont. For more information, please contact scholarship@cuc.claremont.edu. 


\title{
AN ISOMORPHISM THEOREM FOR REAL-CLOSED FIELDS
}

\author{
By P. Erdös, L. Gillman, and M. Henriksen \\ (Received July 6, 1954)
}

A classical theorem of Steinitz [12, p. 125] states that the characteristic of an algebraically closed field, together with its absolute degree of transcendency, uniquely determine the field (up to isomorphism). It is easily seen that the word real-closed cannot be substituted for the words algebraically closed in this theorem. It is therefore natural to inquire what invariants other than the absolute transcendence degree are needed in order to characterize a real-closed field. ${ }^{1}$

For non-denumerable fields, the question is equivalently stated as follows: what invariants in addition to the cardinal number are needed in order to characterize a real-closed field? Now, it is well-known that any two isomorphic realclosed fields are similarly ordered (i.e., as ordered sets). Here we establish the converse implication ${ }^{2}$ for a particular class of non-denumerable, ${ }^{3}$ non-archimedean, real-closed fields. Section 2 of our paper is devoted to the proof of this theorem (Theorem 2.1).

The class of ordered fields to which our isomorphism theorem applies is quite restricted. (In fact, in order that it not be vacuous, we must assume either the continuum hypothesis, or some one of its generalizations to higher cardinals. ${ }^{4}$ ) Nevertheless, we are able to find an application to a class of fields that is not insignificant, namely, those that appear as residue class fields of maximal ideals in rings of continuous functions (on completely regular topological spaces). This discussion is the content of Section 3 , and leads to the theorem that all nonarchimedean residue class fields (the so-called hyper-real fields) of power $\boldsymbol{N}_{1}$ are isomorphic (Theorem 3.5). As a rather interesting corollary to this theorem, we find (using the continuum hypothesis) that all the non-real residue class fields of maximal ideals of a countable complete direct sum of real fields are isomorphic (Corollary 3.9).

Section 4 continues the discussion of non-archimedean residue class fields. The development here leads to the construction of various such fields that arise from the same ring, but have different cardinal numbers (Theorems $4.4 \mathrm{ff}$. and $4.8 \mathrm{ff}$.). (A fortiori, not all such fields that arise from the same ring are isomorphic.) This section is almost entirely set-theoretic in character, and some of the results obtained here have some set-theoretic interest in themselves (Lemmas 4.1 and 4.7). (No use is made of the continuum hypothesis in this section.)

Finally, in Section 5, we pose some unsolved problems.

\footnotetext{
The characteristic of every real-closed field is zero.

${ }^{2}$ Similarity of order implies, of course, equality of cardinal.

3 The converse is false for denumerable fields.

4 Or the existence of the so-called strongly inaccessible cardinals (for definition, see footnote 12).
} 


\section{Preliminary remarks}

In this section, we recall some standard facts from algebra and set-theory. ${ }^{6}$

Every field is an algebraic extension of a pure transcendental extension of its prime field. By the absolute degree of transcendency of a field is meant its transcendence degree over its prime field. The prime field of every ordered field is the rational field $Q$. It follows that for a non-denumerable ordered field, the absolute degree of transcendency coincides with the cardinal number of the field (since an algebraic extension of an infinite field does not increase its cardinal number).

An ordered field $F$ is called real-closed if every positive element of $F$ has a square root in $F$ and every polynomial of odd degree with coefficients in $F$ has a zero in $F$. (The adjunction of a square root of minus one to such a field yields an algebraically closed field.) It is well-known that a real-closed field has a unique ordering (in fact, $a>0$ if and only if $a=b^{2}$ for some non-zero $b$ ). It follows that any isomorphism of a real-closed field is order-preserving.

We shall also need the following important theorem of Artin and Schreier $[17$, p. 232].

TheOREM 1.1 (Artin-Schreier). For every ordered field $F$, there is a unique (up to isomorphism) algebraic extension of $F$ that is real-closed and whose order preserves the order of $F$.

The real-closure of $F$ will be denoted by $a F$.

Turning now to abstract ordered sets, we introduce the following definition, due to Hausdorff (see [4, pp. 180-181]).

Definimion 1.2 (Hausdorff). Let $\alpha$ be any ordinal. A (simply) ordered set $L$ is called an $\eta_{\alpha}$-set provided that:

(i) if $A, B$ are subsets of $L$ of power $<\boldsymbol{N}_{\alpha}$, and such that $A<B,{ }^{6}$ then there is a $y \in L$ with $A<y<B$, and

(ii) no subset $D$ of $L$ of power $\left\langle\boldsymbol{N}_{\alpha}\right.$ is cofinal or coinitial with $L$.

Applying (i) again, there is a $z \in L$ such that $y<z<B$. Hence there is an entire interval of $L$ between $A$ and $B$. Likewise; from (ii), there is an entire interval of $L$ that is $>D$, and an entire interval that is $<D$. With these remarks in mind, the proof of the following lemma becomes evident.

LEMMA 1.3. Every dense subset of an $\eta_{\alpha}$-set is an $\eta_{\alpha}$-set.

The cardinal number of any set $E$ will be denoted by $|E|$.

\section{A characterization theorem for real-closed fields}

In this section, we establish the following theorem.

TheOREM 2.1. Let $\alpha$ be any ordinal $>0$. Any two real-closed fields $F, F^{\prime}$ that are $\eta_{\alpha}$-sets (Definition 1.2) of power $\boldsymbol{\aleph}_{\alpha}$ are isomorphic.

In connection with this theorem, it is known that any two $\eta_{\alpha}$-sets of power

${ }^{5}$ For a general reference in algebra, see [17]; for set-theory, see [4]. The necessary definjtions in connection with rings of functions may be found in [7].

- $A<B(A<y<B)$ means that $a<b(a<y<b)$ for all $a \in A$ and all $b \in B$. 
$\boldsymbol{\aleph}_{\alpha}$ are similar as ordered sets. The set of rationals is an $\eta_{0}$-set (of power $\boldsymbol{\aleph}_{0}$ ). On the other hand, for every ordinal $\beta$, the existence of an $\eta_{\beta+1}$-set of power $\boldsymbol{\aleph}_{\beta+1}$ is equivalent to the validity of the hypothesis $2^{\aleph_{\beta}}=\boldsymbol{\aleph}_{\beta+1}$. For singular $\boldsymbol{\aleph}_{\alpha}$, no $\eta_{\alpha}$-set of power $\aleph_{\alpha}$ can exist [4, pp. 181-182].

To establish our theorem, we first prove two lemmas.

Lemma 2.2. If a subfield $E$ of an ordered field $F$ contains any interval $(a, b)$ of $F$, then $E=F$.

Proof. Let $e_{1}, e_{2}$ be elements of $E$ such that $a<e_{1}<e_{2}<b$. Put $d=e_{2}-e_{1}$. Since $E$ is a field, the closed intervals $I=[0, d]$ and $J=[1 / d, \infty)^{7}$ of $F$ are contained in $E$. Now the interval $K=[d, \infty)$ of $F$ is the translate of $J$ by the element $d-1 / d$ of $E$; therefore $K$, too, is contained in $E$. Thus $E$ contains $I \cup K=$ $[0, \infty)$, i.e., $E$ contains every non-negative element of $F$. It follows that $E=F$.

Lemma 2.3. Every non-denumerable ordered field $F$ has a transcendence base over the rational field $Q$ that is dense in $F$.

Proof. Well-order the set of all intervals $I=(a, b)$ of $F(a, b \in F)$ into a sequence $\left\{I_{\xi}\right\}_{\xi<\omega_{\alpha}}$, where $\boldsymbol{\aleph}_{\alpha}=|\boldsymbol{F}|$. Let $\beta$ be any ordinal $<\omega_{\alpha}$, and suppose that a sequence $\left\{t_{\xi}\right\}_{\xi<\beta}$ of independent transcendentals has been chosen, such that $t_{\xi} \in I_{\xi}$ for each $\xi<\beta$. Let $E_{\beta}$ denote the subfield of $F$ consisting of all elements of $F$ that are algebraic over the field $Q\left(t_{0}, \cdots, t_{\xi}, \cdots\right)_{k<\beta}$. This latter is of power $\left\langle\boldsymbol{N}_{\alpha}\right.$; hence $E_{\beta}$, too, is of power $\left\langle\boldsymbol{N}_{\alpha}\right.$, and therefore $E_{\beta}$ is a proper subfield of $F$. Accordingly, by Lemma $2.2, E_{\beta}$ does not cover any interval of $F$. Hence we may choose a transcendental $t_{\beta} \in I_{\beta}$ that is independent of the set $\left\{t_{\xi}\right\}_{\xi<\beta}$. In this way, we construct a set $S=\left\{t_{\xi}\right\}_{\xi<\omega_{\alpha}}$ of independent transcendentals, with $S$ dense in $F$. We then extend $S$ to a transcendence base for $F$.

Proof of Theorem 2.1. For convenience of notation, we identify the prime fields of $F$ and $F^{\prime}$ with the rational field $Q$. By Lemma 2.3, there exist transcendence bases $T, T^{\prime}$ of $F, F^{\prime \prime}$ that are dense in $F, F^{\prime}$ (resp.). Clearly, $|T|=$ $\left|T^{\prime}\right|=\aleph_{\alpha}$. List the elements of $T$ in a sequence

$$
\left\{t_{\xi}\right\}_{\xi<\omega_{\alpha}}
$$

and list the elements of $T^{\prime}$ in a sequence $\left\{t_{\xi}^{\prime}\right\}_{k<\omega_{\alpha}}$. Let $\beta$ be any ordinal $<\omega_{\alpha}$, and suppose that we have defined a sequence

$$
\left\{s_{\xi}\right\}_{\xi<\beta}
$$

of elements of $T$, and a sequence $\left\{s_{\xi}^{\prime}\right\}_{\xi<\beta}$ of elements of $T^{\prime}$, as follows. Introduce the subfield

$$
E_{\beta}=Q\left(s_{0}, \cdots, s_{\xi}, \cdots\right)_{\xi<\beta}
$$

of $F$, and the subfield $E_{\beta}^{\prime}=Q\left(s_{0}^{\prime}, \cdots, s_{\xi}^{\prime}, \cdots\right)_{\xi<\beta}$ of $F^{\prime}$, and denote their realclosures by

$$
F_{\beta}=\mathcal{R} E_{\beta}, \quad F_{\beta}^{\prime}=\mathfrak{A} E_{\beta}^{\prime} .
$$

\footnotetext{
$7[x, \infty)$ denotes the set of all elements $\geqq x$.
} 
Since $F$ and $F^{\prime}$ are real-closed, we may regard $F_{\beta}, F_{\beta}^{\prime}$ as subfields of $F, F^{\prime}$. Our induction assumption is that there exists an (order-preserving) isomorphism $\Phi_{\beta}$ of $F_{\beta}$ upon $F_{\beta}^{\prime}$.

We now define $s_{\beta}$ and $s_{\beta}^{\prime}$. To simplify the notation, we shall refer to these elements as $x$ and $x^{\prime}$, resp.; furthermore, for any element or subset $A$ of $F_{\beta}$, we shall denote its image $\Phi_{\beta}(A)$ in $F_{\beta}^{\prime}$ by $A^{\prime}$.

There are two cases, according as $\beta$ is even or odd. If $\beta$ is even, we define $x$ to be the element $t_{\xi}$ of (1) of smallest index $\xi$ such that $t_{\xi}$ does not appear in the sequence (2): such an element exists, since the sequence (2) is of power $|\beta|\left\langle\boldsymbol{\aleph}_{\alpha}\right.$. Since $F_{\beta}$ is an algebraic extension of $E_{\beta}$, the transcendental $x$ does not belong to $F_{\beta}$; also, $\left|F_{\beta}\right|=\left|E_{\beta}\right|$. Now from (3), we see that $\left|E_{\beta}\right|\left\langle\boldsymbol{N}_{\alpha}\right.$ (since $\alpha>0$ ); therefore $\left|F_{\beta}\right|<\boldsymbol{N}_{\alpha}$. Finally, the set-union $T^{\prime} \cup F_{\beta}^{\prime}$ is dense in the $\eta_{\alpha}$-set $F^{\prime}$ (since $T^{\prime}$ alone is), and is therefore itself an $\eta_{\alpha}$-set (Lemma 1.3).

We are now prepared to apply the definition of an $\eta_{\alpha}$-set. Decompose the set $F_{\beta}$ into the sets $A_{\beta}, B_{\beta}$, such that $A_{\beta}<x<B_{\beta}$ (one of these sets may be empty). The corresponding subsets $A_{\beta}^{\prime}, B_{\beta}^{\prime}$ of $F_{\beta}^{\prime}$ are both of power $<\boldsymbol{N}_{\alpha}$, and, since $\Phi_{\beta}$ is order-preserving, we have $A_{\beta}^{\prime}<B_{\beta}^{\prime}$. Therefore, by (i) of Definition 1.2, there exists an element $y^{\prime}$ of the $\eta_{\alpha}$-set $T^{\prime} \cup F_{\beta}^{\prime}$ such that $A_{\beta}^{\prime}<y^{\prime}<B_{\beta}^{\prime}$ (or, in case one of the sets of the decomposition is empty, we apply (ii) instead of (i)). We define $x^{\prime}$ to be any such element $y^{\prime}$. Since $A_{\beta}^{\prime} \cup B_{\beta}^{\prime}=F_{\beta}^{\prime}$, we have $x^{\prime} F_{\beta}^{\prime}$; hence

$$
\text { for all } c \in F_{\beta}, x^{\prime}>c^{\prime} \text { if and only if } x>c \text {. }
$$

In case $\beta$ is odd, we proceed analogously, reversing the roles of $T$ and $T^{\prime}$ throughout.

To complete the induction step, we must extend the isomorphism $\Phi_{\beta}$ of $F_{\beta}$ upon $F_{\beta}^{\prime}$ to an isomorphism $\Phi_{\beta+1}$ of $F_{\beta+1}=R E_{\beta}(x)$ upon $F_{\beta+1}^{\prime}=R E_{\beta}^{\prime}\left(x^{\prime}\right)$. Now clearly we have $F_{\beta+1}=\Re F_{\beta}(x), F_{\beta+1}^{\prime}=Q F_{\beta}^{\prime}\left(x^{\prime}\right)$. Accordingly, we begin by extending $\Phi_{\beta}$ to an order-preserving isomorphism $\phi_{\beta+1}$ of $F_{\beta}(x)$ upon $F_{\beta}^{\prime}\left(x^{\prime}\right)$. Define $\phi_{\beta+1}(x)=x^{\prime}$, and then extend $\phi_{\beta+1}$ over $F_{\beta}(x)$ in the natural way (i.e., preserving formal sums and products). Since $x$ and $x^{\prime}$ are transcendentals, $\phi_{\beta+1}$ will be an algebraic isomorphism. Our first problem, then, is to verify that $\phi_{\beta+1}$, thus defined, preserves order.

To this end, it suffices to show that $\phi_{\beta+1}$ preserves order from the polynomial ring $F_{\beta}^{\prime}[x]$ to the ring $F_{\beta}^{\prime}\left[x^{\prime}\right]$ (the extension to the desired quotient fields being then automatic). Thus, we are to show that

$$
f=\sum_{r=0}^{n} a_{r} x^{r}>0 \text { implies } f^{\prime}=\sum_{r=0}^{n} a_{r}^{\prime} x^{\prime^{r}}>0
$$

$\left(a_{r} \in F_{\beta}, a_{n} \neq 0\right.$ ). We may assume $a_{n}=1$. If $n=1$, then (5) holds by construction (ef. (4)). Turning to the case $n=2$, write $f=(x-h)^{2}+k\left(h, k \in F_{\beta}\right)$. If $k \geqq 0$, then trivially $f^{\prime}=\left(x^{\prime}-h^{\prime}\right)^{2}+k^{\prime}>0$. If $k<0$, let $a$ denote the positive square root of $-k$; then $a \in F_{\beta}$, since $F_{\beta}$ is real-closed. Then $f>0$ implies $x<h-a$ or $x>h+a$, whence by (4), $x^{\prime}<h^{\prime}-a^{\prime}$ or $x^{\prime}>h^{\prime}+a^{\prime}$ (resp.), whence $f^{\prime}>0$.

Thus (5) holds for $n=1$ and for $n=2$. Since any polynomial (in one inde- 
terminate) over a real-closed field is the product of linear and quadratic factors, (5) holds in the general case as well. We have thus shown that $\phi_{\beta+1}$ is an orderpreserving isomorphism of $F_{\beta}(x)$ upon $F_{\beta}^{\prime}\left(x^{\prime}\right)$. By the Artin-Schreier theorem (Theorem 1.1), $\phi_{\beta+1}$ has an extension to an (order-preserving) isomorphism $\Phi_{\beta+1}$ between their real-closures, i.e., of $F_{\beta+1}$ upon $F_{\beta+1}^{\prime}$.

This completes the induction step. By alternating in the induction between the cases $\beta$ even and $\beta$ odd, we have ensured that both of the sets $T$ and $T^{\prime}$ will be exhaùsted. Now put

$$
G=\bigcup_{\beta<\omega_{\alpha}} F_{\beta}, \quad G^{\prime}=\bigcup_{\beta<\omega_{\alpha}} F_{\beta}^{\prime} .
$$

It is easily seen that $G$ is real-closed. Obviously, $G \subset F$; and, as just noted, $T \subset G$. Since $F$ is real-closed, and algebraic over $T$, we must have $G=F$. Likewise, $G^{\prime}=F^{\prime}$. But clearly $G$ and $G^{\prime}$ are isomorphic. Therefore $F$ and $F^{\prime}$ are isomorphic. This completes the proof of the theorem.

It is clear that two real-closed fields may be similar as ordered sets and have the same absolute degree of transcendency, and yet not be isomorphic. For example, it is obvious (and well-known) that the smallest real-closed field containing the real number $e$ is not isomorphic with the smallest real-closed field contaning $\pi$. It may be, however, that a non-archimedean real-closed field is characterized by its order type (together with its degree of transcendency over the real field?). We have so far been unable either to prove this, or to find a counter-example.

For examples of non-archimedean real-closed fields of arbitrarily large power that are not $\eta_{\alpha}$-sets, see Sikorski $[10,11]$.

\section{Hyper-real fields of power $\mathfrak{c}=2^{\aleph_{0}}$}

Let $C=C(X)$ denote the ring of all continuous real-valued functions over a completely regular topological space $X{ }^{5}$ Hewitt has shown that if $M$ is any maximal ideal of $C$, then the residue class field $C / M$ is an ordered field that contains the real field $R$. If $C / M$ contains $R$ properly (whence $C / M$ is non-archimedean), then $M$ is called a hyper-real ideal, and $C / M$ is called a hyper-real field. A necessary (but not sufficient) condition that $C$ contain a hyper-real ideal is that $X$ be non-compact [7, Theorem 41].

For every $f \epsilon C(X)$, we write $Z(f)=\{x: x \in X, f(x)=0\} ; Z(f)$ is called the zero-set of $f$. For any ideal $I$ of $C$, we write $Z(I)=\{Z(f): f \in I\}$. It can be seen without difficulty that the family $Z(I)$ is elosed under finite intersection; $M$ is a maximal ideal if and only if $\mathrm{Z}(M)$ is a maximal such family that does not contain the empty set [7, Theorem 36]. An ideal $I$ is called a free ideal if $\bigcap_{f \in I} Z(f)$ is empty. Every hyper-real maximal ideal is free (but not conversely [7, Theorem 41]).

We digress for a moment to insert a definition and a lemma that will be needed at various points in the sequel.

DEFINITION 3.1. By the minimal cardinal associated with a maximal ideal 
$M$, we shall mean the smallest of the cardinal numbers of the dense subsets of $Z$, for $Z \in Z(M)$.

LEMMA 3.2. Let $\mathrm{p}$ denote the associated minimal cardinal of a maximal free ideal $M$. Then $|C(X) / M| \leqq 2^{\mathfrak{p}}$.

Proof. Any two functions that coincide on an element of $Z(M)$ are congruent modulo $M$. Let $Z$ be any element of $Z(M)$ that has a dense subset $Y$ of power $\mathfrak{p}$. Since the values of a continuous function on $Z$ are determined by its values on $Y$, there are at most $c^{\mathfrak{p}}$, i.e., $2^{\mathfrak{p}}$ (since, trivially, $\mathfrak{p}$ is infinite), continuous realvalued functions on $Z$, hence at most this many mutually incongruent such functions.

We return now to the central topic of this section.

Lemma 3.3 If $X$ is any completely regular space, and if $M$ is any hyper-real ideal of $C(X)$, then the residue class field $C(X) / M$ is real-closed, and its degree of transcendency over the real field $R$ is at least $\mathrm{c}$.

Proof. See Henriksen and Isbell [6], Isbell [8], and Hewitt [7, Theorem 42].

For any maximal ideal $M$ of $C$, the residue class modulo $M$ that contains any given $f \in C$ will be denoted by $[f]_{M}-$ or, for brevity, simply by [ $f$ ]. The field $C / M$ is ordered as follows: $[f]>0$ if and only if both (i) the set $\{x: f(x) \geqq 0\}$ is in $\mathrm{Z}(M)$, and (ii) $Z(f) \& \mathrm{Z}(M)[7$, p. 74]. Equivalently: [f] $>0$ if and only if there is a zero-set $Z \in Z(M)$ such that $f(x)>0$ for all $x \in Z$. Thus $M$ is hyper-real if and only if there exists a $\phi \in C$ such that the set $\{x: \phi(x) \geqq n\}$ is in $\mathcal{Z}(M)$ for all $n=1,2, \cdots$.

THeOREM 3.4. If $X$ is any completely regular space, and if $M$ is any hyper-real ideal of $C(X)$, then the residue class field $C(X) / M$ is an $\eta_{1}$-set (Definition 1.2).

Proof. Let $\left\{\left[f_{n}\right]\right\},\left\{\left[g_{n}\right]\right\}(n=1,2, \cdots)$ be two sequences of elements of $C / M$ such that

$$
\left[f_{n}\right]<\left[f_{n+1}\right]<\left[g_{m+1}\right]<\left[g_{m}\right] \quad(m, n=1,2, \cdots) .
$$

In order to verify condition (i) of Definition 1.2 , it is sufficient to find an $h \in C$ such that $\left[f_{n}\right]<[h]<\left[g_{m}\right](m, n=1,2, \cdots)$.

First we note that we may assume, without loss of generality, that

$$
f_{n}(x) \leqq f_{n+1}(x), \text { and } g_{m+1}(x) \leqq g_{m}(x), \text { for all } x \in X
$$

$$
(m, n=1,2, \cdots) \text {. }
$$

For if we put

$$
f_{n}^{\prime}(x)=\max \left\{f_{1}(x), \cdots, f_{n}(x)\right\}, \quad g_{m}^{\prime}(x)=\min \left\{g_{1}(x), \cdots, g_{m}(x)\right\},
$$

for all $x \in X$, then we will have $\left[f_{n}^{\prime}\right]=\left[f_{n}\right]$ and $\left[g_{m}^{\prime}\right]=\left[g_{m}\right]$, by (6) and the definition of the ordering of $C / M$. Obviously $f_{n}^{\prime}(x) \leqq f_{n+1}^{\prime}(x)$ and $g_{m+1}^{\prime}(x) \leqq g_{m}^{\prime}(x)$ for every $x$.

Secondly, we note that we may also assume that

$$
f_{n}(x) \leqq g_{n}(x) \text { for all } x \in X \quad(n=1,2, \cdots) .
$$


For put $f_{1}^{\prime \prime}(x)=f_{1}^{\prime}(x)$, and $g_{1}^{\prime \prime}(x)=\max \left\{f_{1}^{\prime \prime}(x), g_{1}^{\prime}(x)\right\}$. If we have defined $f_{1}^{\prime \prime}, \cdots, f_{n}^{\prime \prime}, g_{1}^{\prime \prime}, \cdots, g_{n}^{\prime \prime}$ so that

$$
f_{1}^{\prime \prime}(x) \leqq \cdots \leqq f_{n}^{\prime \prime}(x) \leqq g_{n}^{\prime \prime}(x) \leqq \cdots \leqq g_{1}^{\prime \prime}(x)
$$

for all $x \in X$, then we put

$$
\begin{aligned}
& f_{n+1}^{\prime \prime}(x)=\min \left\{\max \left\{f_{n}^{\prime \prime}(x), f_{n+1}^{\prime}(x)\right\}, g_{n}^{\prime \prime}(x)\right\}, \\
& g_{n+1}^{\prime \prime}(x)=\max \left\{\min \left\{g_{n}^{\prime \prime}(x), g_{n+1}^{\prime}(x)\right\}, f_{n+1}^{\prime \prime}(x)\right\}
\end{aligned}
$$

(all $x \in X$ ). It follows readily from (6) and the definition of order in $C / M$ that $\left[f_{n}^{\prime \prime}\right]=\left[f_{n}^{\prime}\right]$ and $\left[g_{n}^{\prime \prime}\right]=\left[g_{n}^{\prime}\right]$ for all $n=1,2, \cdots$. Moreover, we have

$$
f_{n}^{\prime \prime}(x) \leqq f_{n+1}^{\prime \prime}(x) \leqq g_{n+1}^{\prime \prime}(x) \leqq g_{n}^{\prime \prime}(x)
$$

for all $x \in X$.

Resuming our original notation, we assume that (6), (7) and (8) hold. Since $M$ is hyper-real, there is a function $\phi \in C$ such that for all $x \in X$, we have $\phi(x) \geqq 1$, and, for every $n=1,2, \cdots$, the set

$$
\Phi_{n}=\{x: \phi(x) \geqq n\}
$$

is in $\mathcal{Z}(M)$. We now define a function $h$ as follows: $:^{8}$

$$
\begin{aligned}
h(x)=(n+1-\phi(x)) f_{n}(x)+ & (\phi(x)-n) f_{n+1}(x) \\
& \text { whenever } n \leqq \phi(x) \leqq n+1(n=1,2, \cdots) .
\end{aligned}
$$

Evidently, $h \in C(X)$. Clearly, $f_{n}(x) \leqq h(x) \leqq f_{n+1}(x)$ whenever $n \leqq \phi(x) \leqq n+1$. Since the sequence of functions $\left\{f_{n}\right\}$ is monotone increasing (7), we have $h(x) \geqq$ $f_{n+1}(x)$ for all $x \epsilon \Phi_{n+1}$. Now by (6), there is a $Z_{n+1} \in Z(M)$ such that $f_{n+1}(x)>$ $f_{n}(x)$ for all $x \in Z_{n+1}$. Therefore $h(x)>f_{n}(x)$ for all $x$ in the set $\Phi_{n+1} \cap Z_{n+1}$ which is in $Z(M)$ (since $Z(M)$ is closed under finite intersection). Thus $[h]>\left[f_{n}\right]$ for all $n=1,2, \cdots$.

Next, it follows from (7) and (8) that for each fixed $m, f_{m+p}(x) \leqq g_{m+1}(x)$ for all $x \in X$ and all $p=1,2, \cdots$. It is easily seen from this that $h(x) \leqq g_{m+1}(x)$ for all $x \in \Phi_{m}$. As above, there is a $Z_{m}^{\prime} \in \mathbb{Z}(M)$ such that $g_{m+1}(x)<g_{m}(x)$ for all $x \in Z_{m}^{\prime}$, whence $h(x)<g_{m}(x)$ for all $x \in\left(Z_{m}^{\prime} \cap \Phi_{m}\right) \in Z(M)$, i.e., $[h]<\left[g_{m}\right]$. We have thus verified condition (i) of Definition 1.2. Since the definition of $h$ depended only upon the $f_{n}$ 's-not upon the $g_{m}$ 's-the arguments just given yield the additional fact that $C / M$ has no countable cofinal subset (and hence no countable coinitial subset). ${ }^{8}$ Thus we have also verified (ii) of Definition 1.2. This completes the proof that $C / M$ is an $\eta_{1}$-set.

Theorem 3.5. Under the continuum hypothesis $\mathfrak{c}=\boldsymbol{\aleph}_{\mathbf{1}}$, all hyper-real fields of power $\mathrm{c}$ are isomorphic.

Proof. These fields are $\eta_{1}$-sets (Theorem 3.4) of power $\boldsymbol{\aleph}_{1}$, and are real-closed (Lemma 3.3). Hence, by Theorem 2.1, they are all isomorphic.

${ }^{8}$ We are indebted to J. R. Isbell for the device used here, which was used by Isbell to show that $C / M$ has no countable cofinal subset [9] (written communication). 
Another way of expressing this theorem is: all hyper-real fields of power $\aleph_{1}$ are isomorphic.

CoRollaRY 3.6. If $|C(X)|=\aleph_{1}$, then all the hyper-real fields $C(X) / M$ are isomorphic.

CoRoLlary 3.7. If $\mathfrak{c}=\boldsymbol{N}_{1}$, then all the hyper-real fields $C(X) / M$ for which some $Z \in Z(M)$ has a denumerable dense subset are isomorphic.

Proof. Lemma 3.2.

CoROLLARY 3.8. If $\mathfrak{c}=\boldsymbol{\aleph}_{1}$, and if $X$ is separable metric or denumerable, then all the hyper-real fields $C(X) / M$ are isomorphic.

CoRollary 3.9. If $\mathfrak{c}=\boldsymbol{\aleph}_{1}$, then all the non-real residue class fields of maximal ideals of a denumerable complete direct sum of real fields are isomorphic.

Proor. The direct sum in question may be identified with the ring of all (continuous) real-valued functions on the denumerable discrete space.

If it were true that every $Z(M)$ contained a discrete set, then the proof of Theorem 3.4 could be considerably simplified. Unfortunately, this is not true even for the case $X=R$ (the real line), as is shown by the following example due to W. F. Eberlein [5].

EXAMPLE 3.10. Let $\Gamma$ be the family of all closed subsets of $R$ whose complements have finite (Lebesgue) measure. Let $I=\{f: f \epsilon C(R), Z(f) \in \Gamma\}$. It is easily seen that $I$ is a proper ideal of $C(R)$. Let $M$ be any maximal ideal containing $I$. It is easily verified that $M$ is hyper-real (see also [7, Theorem 53]). We shall show that $Z(M)$ contains no discrete set. In fact, $Z(M)$ contains no set of finite measure. For suppose that $Z \in \mathrm{Z}(M)$ has finite measure. Then there is an open set $V$ of finite measure contaning $Z$. But then we have $R-V \in \Gamma \subset Z(M)$ contrary to the fact that $Z(M)$ is closed under finite intersection but does not contain the empty set.

A consequence of this construction is the existence of a point $p$ in the StoneCech compactification $[13,1]$ of $R$ such that every neighborhood of $p$ intersects $R$ in a set of infinite measure (see $[2 ; 3$, Theorem 1]).

\section{Cardinal numbers of hyper-real fields}

In this section, we establish some theorems concerning the cardinal numbers of various hyper-real fields. The continuum hypothesis is not used (except in a remark at the end of the section). The purely set-theoretic results that are used in the proofs have some interest for their own sake, and, accordingly, are stated separately as lemmas.

Lemma 4.1. ${ }^{9}$ For every set $X$ of infinite power $\mathfrak{m}$, there exists a set of more than $\mathfrak{m}$ subsets of $X$, each of power $\mathrm{m}^{10}{ }^{10}$ and such that the intersection of any two of them is of power <m.

Proof. Express $X$ as the union of $\mathfrak{m}$ mutually disjoint sets $S_{\alpha}\left(\alpha<\omega_{\mathrm{m}}\right)^{11}$

${ }^{9}$ This lemma was proved by P. Erdös in 1934, but has not been published heretofore. For a large collection of results on almost disjoint ssts, see Tarski [14, 15].

${ }^{10}$ In our application of this lemma, we will not need the fact that the sets are of power m.

${ }^{11}$ Here (and subsequently) we use the symbol $\omega_{m}$ to denote the least ordinal of power $\mathrm{m}$. 
each of power $m$. Well-order each $S_{\alpha}$ in a sequence of type $\omega_{\mathrm{m}}$, and, for each $\beta<\omega_{\mathrm{m}}$, form the set $A_{\beta}$ consisting of all the elements of index $\beta$ (one from each set $S_{\alpha}$ ). Then the collection $P$ of the $m$ sets $A_{\beta}$ has the following properties: (i) each $A \in P$ is of power m, (ii) each $A \in P$ meets every $S_{\alpha}$ in exactly one element, and (iii) the intersection of any two distinct sets of $P$ is of power $<\mathrm{m}$. (In fact, the intersection is empty.) By the maximal chain theorem of Hausdorff (or Zorn's lemma), there is a maximal family $Q$ containing $P$ and satisfying (i), (ii) and (iii). We shall show that $|Q|>\mathfrak{m}$, which will establish the theorem. In the contrary case, $|Q|=\mathrm{m}$ (since $Q \supset P$ ), and the elements of $Q$ can accordingly be enumerated in a sequence $\left\{B_{\beta}\right\}_{\beta<\omega_{m}}$. We define a new set $B$ as follows. From (ii), we see that for every $\alpha<\omega_{\mathrm{m}}$, the intersection of $S_{\alpha}$ with the set $\mathrm{U}_{\beta<\alpha} B_{\beta}$ is of power $<\mathrm{m}$. Hence, since $\left|S_{\alpha}\right|=\mathrm{m}$, the set $S_{\alpha}-\mathrm{U}_{\beta<\alpha} B_{\beta}$ is not empty. We define $x_{\alpha}$ to be any arbitrary element of this latter set. The set $B=\left\{x_{\alpha}\right\}_{\alpha<\omega_{m}}$, thus defined, clearly satisfies (i) and (ii). To verify (iii), we observe that for each $\alpha<\omega_{m}$, we have $B \cap B_{\alpha} \subset\left\{x_{\beta}\right\}_{\beta \leqq \alpha}$; therefore $\left|B \cap B_{\alpha}\right|<\mathfrak{m}$. Thus $Q$ was not maximal, as supposed. Accordingly, we must have $|Q|>\mathrm{m}$.

As already noted, if $M$ is any maximal free ideal of a ring $C(X)$, then the set $Z(M)$ is a maximal subset of $Z(C)$ that is closed under finite intersection, does not contain the empty set, and has total intersection void. If $X$ is discrete, then, of course, $Z(C)$ is the set of all subsets of $X$. In discussing this case, it has often been found convenient to speak in terms of a non-trivial, finitely additive, two-valued measure $\mu$ on the set $X$; by this we mean: $\mu$ is defined on all subsets of $X$, to each it assigns the value 0 or 1 , every one-element set has measure 0 , the entire set $X$ has measure $1, \mu$ is finitely additive. Thus (if $X$ is discrete) there is a natural one-one correspondence between the set of all such two-valued measures $\mu$ on $X$ and the set of all maximal free ideals $M$ of $C$-under which $\mu(Z)=1$ if and only if $Z \in \mathrm{Z}(M)$.

Let us call a cardinal $\mathfrak{m}$ nonmeasurable if there exists no non-trivial, countably additive, two-valued measure on a set of power $\mathfrak{m}$. Every $\mathfrak{m}$ smaller than the first strongly inaccessible cardinal is nonmeasurable. ${ }^{12}$ Now for any discrete space $X$, a maximal free ideal $M$ of $C(X)$ is hyper-real if and only if the finitely additive measure $\mu$ corresponding to $M$ fails to be countably additive [7, Theorem 50]. Therefore, if the cardinal of the discrete space $X$ is nonmeasurable, the maximal free ideals of $C(X)$ coincide with the hyper-real ideals. In particular, this is the case whenever $|X| \leqq c$.

Lemma 4.2. Let $X$ be any set, and suppose that $X$ has a subset $Y$ of infinite power $n$. Then there exists a non-trivial, finitely additive, two-valued measure $\mu$ on $X$ such that $\mu(Y)=1$, and every subset of $X$ of power $<\mathfrak{n}$ is of measure zero-and if $\mathrm{n}$ is nonmeasurable, then $\mu$ is not countably additive.

Proof. Suppose first that $Y=X$. Let $P$ be the set of all subsets $S$ of $X$ whose

12 This result is due to Ulam and Tarski. (A regular cardinal $m>\boldsymbol{N}_{0}$ is strongly inaccessible if $2^{\mathfrak{n}}<\mathrm{m}$ for all $\mathfrak{n}<\mathrm{m}$. No such cardinal is known.) See [16]. 
complements are of power $<\mathfrak{n}$. Then $P$ is closed under finite intersection. Embed $P$ in a maximal system $Q$ that is closed under finite intersection, and that excludes the empty set. Obviously, every element of $Q$ is of power $\pi$. Define $\mu(E)=1$ if and only if $E \in Q$. If $\mathfrak{n}$ is nonmeasurable, then by definition, $\mu$ cannot be countably additive.

Applying this result to the general case, let $\mu_{Y}$ be a non-trivial, finitely additive, two-valued measure defined on $Y$ such that every subset of $Y$ of power $<$ n is of measure 0. Extend $\mu_{Y}$ to a two-valued measure $\mu$ on $X$ as follows: for $A \subset X$, $\mu(A)=\mu_{Y}(A \cap Y)$. It is easily verified that the measure $\mu$ has the required properties (cf. [16, p. 146]).

As a particular application of this lemma, we have:

CoRoLhary 4.3. For every infinite discrete space $X$, there exist hyper-real ideals $M$ of $C(X)$ for which $|C(X) / M|=\mathfrak{c}$.

Proor. By Lemma 4.2, there exist hyper-real ideals whose associated minimal cardinal (Definition 3.1) is $\boldsymbol{\aleph}_{0}$. The result now follows from Lemma 3.2.

THeORem 4.4. Let $X$ be the discrete space of power c. Then for every hyper-real ideal $M$ of $C(X)$ for which every $Z \in Z(M)$ is of power $\mathrm{c}^{13}$ we have $|C(X) / M|>\mathrm{c}$.

Proor. By Lemma 4.1, there is a set $\left\{A_{i}\right\}_{i \in I}$ of more than $\mathfrak{c}$ subsets of $X$, the intersection of any two of which is of power <c. Let $\phi$ be any element of $C(X)$ that assumes no value twice (i.e., $\phi$ is a one-one mapping of $X$ into the reals). For each $i \in I$, define $f_{i}$ as any function on $X$ that assumes precisely the values $\phi\left(A_{i}\right)$, i.e., $f_{i}(X)=\phi\left(A_{i}\right)$. Then $f_{i}(x)=f_{j}(x)=r$ only if $r \epsilon \phi\left(A_{i}\right) \cap \phi\left(A_{j}\right)$, hence only if $x \in A_{i} \cap A_{j}$. Consequently, if $i \neq j, f_{i}$ agrees with $f_{j}$ only on a set of power $<\mathfrak{c}$-therefore on no set $Z \in \mathbb{Z}(M)$. Thus $f_{i} \not \equiv f_{j}(\bmod M)$. Hence $\left\{f_{i}\right\}_{i \in t}$ is a set of more than $c$ mutually incongruent $(\bmod M)$ elements of $C(X)$.

CoRoLLARY 4.5. The hyper-real fields associated with the discrete space of power $\mathfrak{c}$ do not all have the same cardinal number-a fortiori, they are not all isomorphic.

Proof. Corollary 4.3 and Theorem 4.4.

LEMMA 4.6. Let $\left\{E_{\beta}\right\}_{\beta<\omega_{\mathfrak{n}}}$ be a family of $\mathfrak{n}$ sets of power $\mathfrak{m}$, where $\mathfrak{m}$ is infinite, and $0<\mathfrak{n} \leqq \mathfrak{m}$. Then there is a set $\left\{H_{\beta}\right\}_{\beta<\omega_{\mathfrak{n}}}$ of mutually disjoint sets of power $\mathrm{m}$, with $H_{\beta} \subset E_{\beta}$ for all $\beta<\omega_{\pi}$.

Proof. By repeating the set $E_{0} \mathfrak{m}$ times, we can secure the condition $\mathfrak{n}=\mathfrak{m}$. Let $\alpha$ be any ordinal $<\omega_{m}$, and $\beta$ any ordinal $\leqq \alpha$, and suppose that a set of distinct elements

$$
K_{\alpha, \beta}=\left\{x_{\sigma, r}\right\}_{\sigma<\alpha, \tau \leqq \sigma} \cup\left\{x_{\alpha, \xi}\right\}_{\xi<\beta}
$$

has been defined, such that every $x_{\alpha, \tau} \in E_{\tau}$, and every $x_{\alpha, \xi} \in E_{\xi}$. Then $\left|K_{\alpha, \beta}\right|<\mathfrak{m}$; we choose $x_{a, \beta}$ as any element of $E_{\beta}-K_{\alpha, \beta}$. We have thus defined a set of distinct elements $\left\{x_{\alpha, \beta}\right\}_{\alpha<\omega_{\mathrm{m}}, \beta \leqq \alpha}$, with every $x_{\alpha, \beta} \in E_{\beta}$. We put $H_{\beta}=\left\{x_{\alpha, \beta}\right\}_{\beta \leqq \alpha<\omega_{\mathrm{m}}}$.

LEMMA 4.7. Let $X$ be any set of infinite power $\mathrm{m}$, and let $\mathfrak{q}$ be any infinite cardinal

13 The existence of such ideals follows from Lemma 4.2. 
$\leqq \mathfrak{m}$. Then there exists a system $K=\left\{X_{a, s}\right\}_{a \in A, z \in S}$ of subsets of $X$, where $|A|=$ $\mathfrak{q}$ and $|S|>\mathfrak{m}$, such that

$$
\begin{gathered}
\text { for all } s \in S, \quad \cup_{a \in A} X_{a, s}=X ; \\
\text { for all } s \in S, \text { and for all } a, b \in A, \text { with } a \neq b, \\
X_{a, b} \cap X_{b, s} \text { is empty; }
\end{gathered}
$$

for every $s, t \in S$, with $s \neq t$, put

$$
Y_{8, t}=\bigcup_{a \in A}\left(X_{a, 8} \cap X_{a, t}\right)
$$

then

(11) every subset $E$ of $X$ that is the complement of the union of a finite number of the sets $Y_{s, t}$ is of power $\mathrm{m}$.

Proof. Certainly such a system exists for $|A|=q$ with $|S|=2$. By the maximal chain theorem, there is a maximal system $K$ for $|A|=\mathfrak{q}$; we shall show that for such a $K$ (for which, clearly, $|S| \geqq 2$ ), we have $|S|>\mathfrak{m}$.

Suppose, on the contrary, that $|S| \leqq \mathrm{m}$. We shall find that then $K$ is not maximal. Let $P$ denote the set of all finite subsets of the set of all two-element subsets $\{s, t\}$ of $S$. Then $|P| \leqq \mathrm{m}$. Each $p \in P$ determines a set $E_{p}=X-$ $\bigcup_{\{s, t\} \in p} Y_{s, t}$ as in (11). Conversely, to each set $E$ as in (11), there corresponds at least one $p \in P$ for which $E=E_{p}$. By Lemma 4.6 , there is a set $\left\{H_{p}\right\}_{p \in P}$ of mutually disjoint sets of power $\mathrm{m}$, with each $H_{p} \subset E_{p}$.

Now let $u$ be any index ${ }_{\phi} S$; we construct a set of sets $\left\{X_{a . u}\right\}_{a \in A}$, as follows. Let $x$ be any element of $X$. If $x$ is in no $H_{p}$, we assign $x$ to an arbitrary $X_{a, u}$. In the contrary case, there is exactly one $p$ for which $x \in H_{p}$. Let

$$
S_{p}=U_{\{s, t\} \in p}\{s, t\} .
$$

For each $s \in S_{p}$, there is, by (9) and (10), exactly one $a_{z} \in A$ such that $x \in X_{a_{s}, s}$. Let $a(p)$ be any element of the infinite set $A$ distinct from each of the finitely many elements $a_{s}\left(s \in S_{p}\right)$; we assign $x$ to the set $X_{a(p), u}$.

Write $S^{\prime}=S \cup\{u\}$. Clearly, the enlarged system $K^{\prime}=\left\{X_{a, 8}\right\}_{a \epsilon A, \varepsilon \in S^{\prime}}$ satisfies (9) and (10) (with $S$ therein replaced by $S^{\prime}$ ). To verify (11), let $p^{\prime}$ be any finite subset of the set $P^{\prime}$ of all two-element subsets of $S^{\prime}$; we are to show that $\left|E_{p^{\prime}}\right|=$ $\mathfrak{m}$. Since $|S| \geqq 2$, there is a $p^{\prime \prime} \in P^{\prime}$ that contains $p^{\prime}$ and that also contains at least one element of $P$; since $E_{p^{*}} \subset E_{p^{\prime}}$, it suffices to show that $\left|E_{p^{*}}\right|=\mathfrak{m}$. Let $p$ be the element of $P$ consisting of all the two-element subsets of the set

$$
T_{p^{*}}=\bigcup_{\{s, t\} \in p^{*}}\{s, t\} \cap S ;
$$

then $p$ is not empty (by definition of $p^{\prime \prime}$ ). The set $H_{p}$ is contained in $E_{p}$. Furthermore, by the construction above, we have $H_{p} \subset X_{a(p), u}$, whence none of the sets $Y_{s, u}\left(s \in T_{p^{\prime \prime}}\right)$ meets $H_{p}$. It follows that $H_{p} \subset E_{p^{\prime \prime}}$. Since $\left|H_{p}\right|=\mathfrak{m}$, we have $\left|E_{p^{\prime \prime}}\right|=\mathrm{m}$. As indicated above, this implies that the system $K^{\prime}$ satifies (11). Thus $K^{\prime}$ satisfies (9), (10) and (11). Therefore $K$ was not maximal, as supposed. Accordingly, we have $|S|>\mathrm{m}$. 
Theorem 4.8. Let $X$ be the discrete space of cardinal $\mathfrak{m} \geqq \mathfrak{c}$. Then there exists a hyper-real ideal $M$ of $C(X)$ for which $|C(X) / M|>\mathfrak{m}$.

Proof. Let the system $K$ be as in Lemma 4.7 , with $q=c$. The set of all finite unions $F$ of sets $Y_{s . t}$ is closed under finite union; hence if we define $\mu=0$ on all subsets of all such $F$, and $\mu=1$ elsewhere, then $\mu$ will be a non-trivial, finitely additive, two-valued measure on $X$. Let $M$ be the maximal free ideal corresponding to the measure $\mu$. Let the set of all reals be indexed $\left\{r_{a}\right\}_{a \in A}$. For every $s \in S$, we define a function $f_{s} \in C(X)$ as follows: given any $x \in X$, we have $x \in X_{a, s}$ for exactly one $a \in A$-we put $f_{s}(x)=r_{a}$. Then, for $s \neq t$, we have $f_{s}(x)=f_{t}(x)=r_{a}$ if and only if $x \in X_{a, s} \cap X_{a, t}$. Therefore the set of all $x$ on which $f_{s}$ agrees with $f_{t}$ is the set $Y_{s, t}$. Since $\mu\left(Y_{s, t}\right)=0$, we have $f_{s} f_{t}(\bmod M)$. Thus $\left\{f_{s}\right\}_{s \in s}$ is a set of more than $m$ mutually incongruent $(\bmod M)$ elements of $C$. Accordingly, $|C(X) / M|>\mathrm{m}$. And, since $\mathrm{m} \geqq \mathfrak{c}, M$ must be hyper-real.

Observe that for the special case $\mathfrak{m}=\mathfrak{c}$, a stronger conclusion is given by Theorem 4.4.

Corollary 4.9. Let $X$ be the discrete space of cardinal $\mathfrak{m} \geqq \mathfrak{c}$, and let $\mathfrak{n}$ be any cardinal such that $\mathfrak{m} \geqq \mathfrak{n} \geqq \mathfrak{c}$. Then there exists a hyper-real ideal $M$ of $C(X)$ such that $\mathfrak{n}<|C(X) / M| \leqq 2^{\mathfrak{n}}$.

Proof (ef. proof of Lemma 4.2). Let $Y$ be any subspace of power $n$. By Theorem 4.8, there exists a maximal free ideal $M_{Y}$ of $C(Y)$ for which $\left|C(Y) / M_{Y}\right|$ $>$ n. Define

$$
M=\left\{f: f \in C(X), \quad(Z(f) \cap Y) \in \mathbb{Z}\left(M_{Y}\right)\right\} .
$$

It is verified without difficulty that $M$ is a maximal free ideal of $C(X)$. With each $f_{Y} \in C(Y)$, associate the function $f_{0} \epsilon C(X)$ that agrees with $f_{Y}$ on $Y$ and vanishes everywhere on $X-Y$. Clearly $f_{0} \equiv g_{0}(\bmod M)$ if and only if $f_{Y} \equiv g_{Y}(\bmod$ $\left.M_{Y}\right)$. Hence $|C(X) / M| \geqq\left|C(Y) / M_{Y}\right|$; therefore $|C(X) / M|>\mathfrak{n} \geqq \mathfrak{c}$, and $M$ is hyper-real. To establish the remaining inequality, we observe that $Z(M)$ contains the set $Y$, which is of power $n$. Hence, from Lemma $3.2,|C(X) / M| \leqq 2^{n}$.

If we assume the generalized continuum hypothesis, then in Theorem 4.4 we can conclude that $|C / M|=2^{\mathrm{c}}$, and, in Theorem 4.8 , that $|C / M|=2^{\mathrm{m}}$; and in Corollary 4.9 , we will have that for every $\beta \leqq \alpha$, where $\boldsymbol{\aleph}_{\alpha}=\mathfrak{m}$, there exists a hyper-real ideal $M$ with $|C / M|=\boldsymbol{N}_{\beta+1}$.

\section{Some open problems}

5.1. Is a non-denumerable real-closed field--in particular, if it is non-archimedean-characterized by its type of order as an ordered set? (See the remarks at the close of Section 2.)

5.2. Are all hyper-real fields of the same power isomorphic? (This would follow from Theorem 2.1 if every hyper-real field of power $\boldsymbol{\aleph}_{\alpha}$ were an $\eta_{\alpha}$-set (Definition 1.2). On the other hand, this latter would imply that no hyper-real field of singular cardinal can exist-is this true?)

5.3. Let $m$ denote the minimal cardinal associated with the hyper-real ideal 
$M$ (Definition 3.1); is the field $C / M$ necessarily of power $>\mathfrak{m}$ ? (We know this is true if $\mathfrak{m}=\boldsymbol{\aleph}_{0}$, or if $X$ is discrete and $\mathfrak{m}=\mathrm{c}$.)

5.4. What, if any, of the various results that are obtained using the continuum hypothesis or its extensions, can be derived without the intervention of these hypotheses? Specifically, are any two real-closed fields-in particular, any two hyper-real fields-that are $\eta_{1}$-sets of power $c$, necessarily isomorphic?

UNiversity OF Notre DAME

PuRdue UnIVẹrsity

\section{BibliogRAPHY}

1. E. Свсн, On bicompact spaces, Ann. of Math., 38 (1937), 823-844.

2. I. Gelfand and A. N. KolmogorofF, On rings of continuous functions on topological spaces, C. R. (Doklady) Acad. Sci. URSS., 22 (1939), 11-15.

3. L. Gillman, M. Hentiksen, and M. Jerison, On a theorem of Gelfand and Kolmogoroff concerning maximal ideals in rings of continuous functions, Proc. Amer. Math. Soc., 5 (1954), 447-455.

4. F. Hausdonfr, Grundzüge der Mengenlehre, Leipzig, 1914.

5. M. HENRIKSEN, On the maximal ideals of the ring of entire functions and other function rings, Thesis, University of Wisconsin, 1951 (unpublished).

6. M. HENRIKSEN and J. R. IsBELL, On the continuity of the real roots of an algebraic equation, Proc. Amer. Math. Soc., 4 (1953), 431-434.

7. E. Hewrt, Rings of real-valued continuous functions I, Trans. Amer. Math. Soc., 64 (1948), 45-99.

8. J. R. Isbel, More on the continuity of the real roots of an algebraic equation, Proc. Amer. Math. Soc., 5 (1954), 439.

9. J. R. IsBell, Some details concerning function rings, Bull. Amer. Math. Soc., 60 (1954), abstract 373t, 259-260.

10. R. Sikonski, On an ordered algebraic field, Soc. Sei. Lett. Varsovie C. R. Cl. III Sci. Math. Phys., 41 (1948), 69-96 (1950).

11. R. Sikorsix, On algebraic extensions of ordered fields, Ann. Soc. Polon. Math., 22 (1949), 173-184 (1950).

12. E. Steinitz, Algebraische Theorie der Körper, Berlin, 1930.

13. M. H. Stone, Applications of the theory of Boolean rings to general topology, Trans. Amer. Math. Soc., 41 (1937), 375-481.

14. A. TARSKx, Sur la décomposition des ensembles en sous-ensembles presque disjoints, Fund. Math., 12 (1928), 188-205.

16. A. Takski, Sur la décomposition des ensembles en sous-ensembles presque disjoints, Fund. Math., 14 (1929), 205-215.

16. S. Ulam, Zur Masstheorie in der allgemeinen Mengenlehre, Fund. Math., 16 (1930), 140-150.

17. B. L. van DER WAERden, Moderne Algebra, I, Berlin, 1930. 